\begin{tabular}{|c|c|}
\hline Title & $\begin{array}{l}\text { Superior fracture resi stance of fiber reinforced polyampholyte hy drogels achieved by extraordinarily large energy- } \\
\text { dissi pative process zones }\end{array}$ \\
\hline Author(s) & $\begin{array}{l}\text { Huang, Y iwan; King, Daniel Rudolf; Cui, Wei; Sun, T aolin; Guo, Honglei; Kurokawa, Takayuki; Brown, Hugh R.; } \\
\text { Hui, Chung Y uen; Gong, Jian Ping }\end{array}$ \\
\hline Citation & $\begin{array}{l}\text { Journal of material s chemistry A, Material s for energy and sustainability, 7(22), 13431-13440 } \\
\text { https://doi.org/10.1039/C9T A 02326G }\end{array}$ \\
\hline Issue Date & 2019-06-14 \\
\hline Doc URL & http:/hdl .handle.net/2115/78710 \\
\hline Tyре & article (author version) \\
\hline File Information & JMatterChemA (2019)_Huang.pdf () \\
\hline
\end{tabular}

Instructions for use 


\section{Journal of \\ Materials Chemistry A}

\section{Accepted Manuscript}

This article can be cited before page numbers have been issued, to do this please use: Y. Huang, D. R. King, W. Cui, T. Sun, H. Guo, T. Kurokawa, H. R. Brown, C. Y. Hui and J. P. Gong, J. Mater. Chem. A, 2019, DOI: 10.1039/C9TA02326G.

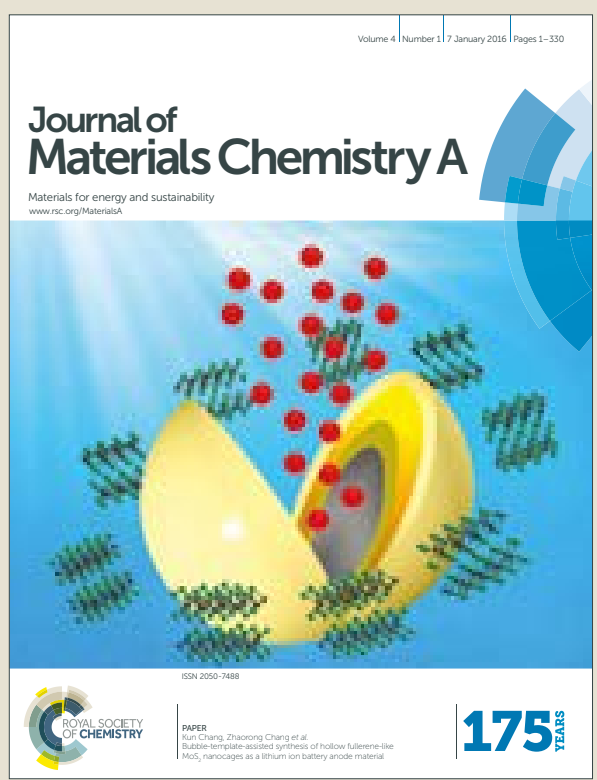

This is an Accepted Manuscript, which has been through the Royal Society of Chemistry peer review process and has been accepted for publication.

Accepted Manuscripts are published online shortly after acceptance, before technical editing, formatting and proof reading Using this free service, authors can make their results available to the community, in citable form, before we publish the edited article. We will replace this Accepted Manuscript with the edited and formatted Advance Article as soon as it is available.

You can find more information about Accepted Manuscripts in the author guidelines.

Please note that technical editing may introduce minor changes to the text and/or graphics, which may alter content. The journal's standard Terms \& Conditions and the ethical guidelines, outlined in our author and reviewer resource centre, still apply. In no event shall the Royal Society of Chemistry be held responsible for any errors or omissions in this Accepted Manuscript or any consequences arising from the use of any information it contains. 


\title{
Superior Fracture Resistance of Fiber Reinforced Polyampholyte Hydrogels Achieved by Extraordinarily Large Energy-dissipative Process Zones $\dagger$
}

Yiwan Huang, ${ }^{a}$ Daniel R. King, ,a,b Wei Cui, ${ }^{c}$ Tao Lin Sun, ${ }^{a, b}$ Honglei Guo, ${ }^{\text {a,b }}$ Takayuki

Kurokawa, ${ }^{\mathrm{a}, \mathrm{b}}$ Hugh R. Brown, ${ }^{\mathrm{e}}$ Chung-Yuen Hui, ${ }^{\mathrm{f}}$ Jian Ping Gong ${ }^{*}, \mathrm{a}, \mathrm{b}, \mathrm{d}$

${ }^{a}$ Faculty of Advanced Life Science, Hokkaido University, Sapporo 001-0021, Japan

${ }^{b}$ Soft Matter GI-CoRE, Hokkaido University, Japan

${ }^{c}$ Graduate School of Life Science, Hokkaido University, Sapporo 001-0021, Japan

${ }^{d}$ Institute for Chemical Reaction Design and Discovery (WPI-ICReDD), Hokkaido University, N21W10, Kita-ku, Sapporo 001-0021, Japan

e ARC Centre of Excellence for Electromaterials Science and Australian Institute for Innovative Materials, University of Wollongong, Innovation Campus, Squires Way, North Wollongong, NSW, 2522, Australia

${ }^{f}$ Department of Mechanical and Aerospace Engineering, Field of Theoretical and Applied Mechanics, Cornell University, Ithaca, NY 14853, USA

*E-mail: gong@sci.hokudai.ac.jp

$\dagger$ Electronic Supplementary Information (ESI) available. See DOI: 10.1039/x0xx00000x

\section{Abstract}

Fiber reinforced soft composites (FRSCs) have been developed recently by combining tough but soft polyampholyte hydrogels with stiff yet flexible woven glass fabrics. In this work, we find that the soft composites show increased tearing resistance with sample size and achieve size-independent, exceptionally high tearing energy above a specific size on the centimeter scale. Such size-dependent tearing behavior correlates with the failure mode change from fiber pull-out to fiber fracture. These findings demonstrate that the rigid fibers in the soft matrices transmit force over a large distance, giving the composites very large process zones. Tremendous energy is dissipated in the large process zones, resulting in the superior fracture
\end{abstract}


resistance of FRSCs. By saturation of the process zone size, the soft composites bediditatede Online extraordinarily tough, showing an intrinsic tearing energy of $\sim 1000 \mathrm{~kJ} \mathrm{~m}^{-2}$ that outperforms other existing tough materials. These novel FRSC materials from hydrated biocompatible hydrogels fill the gap between soft materials and traditional rigid materials, as demonstrated by their high tensile modulus (several GPa) and strength (> $300 \mathrm{MPa}$ ), along with exceptionally high tearing toughness.

Keywords: fiber reinforced hydrogels, super tough hydrogels, material failure modes, energydissipative process zone, fracture model

\section{Introduction}

Fiber reinforced polymers (FRPs), made up of a polymer matrix reinforced with stiff fibers, have been developed for applications which require high strength. ${ }^{1,2}$ While traditional FRPs provide high stiffness and strength, they show limited crack resistance. In general, relatively stiff polymer matrices have been utilized in FRPs. For example, glass fiber reinforced epoxy resins have a matrix of $\sim \mathrm{GPa}$ modulus. The lack of highly efficient energy dissipation mechanisms in such hard-hard combinations might account for the relatively poor crack resistance of these composites. In contrast, fibrous load-bearing soft tissues, such as ligaments and tendons, possess both excellent mechanical strength and high damage resistance. ${ }^{3-5}$ These biological tissues have composite structures that are made up of highly stiff collagen fibers embedded in viscoelastic soft biological matrices, and thus are quite different from the hardhard combinations used in traditional FRPs. These biological systems inspired us to design new composites by incorporating energy-dissipative soft matrices with a stiff fiber reinforcing phase. Recently, we have developed hydrogel composites by embedding stiff yet flexible woven glass fabrics in tough and viscoelastic polyampholyte (PA) hydrogels. ${ }^{6,7}$ The hydrogel composites have exhibited extraordinary mechanical performance (especially tearing energy) that far exceeds the simple combination of the individual components, indicating a strong synergistic 


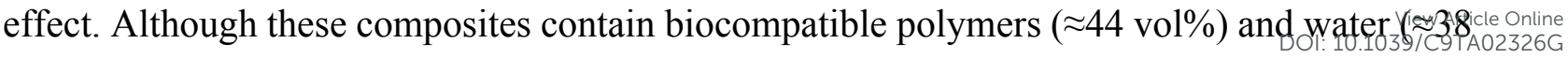
vol\%), ${ }^{7,8}$ they are still mechanically comparable to elastomer composites, such as Kevlar/polyurethane blends. ${ }^{9}$ These results are important not only for developing soft biological prosthetics, but more generally for other applications such as tear-resistant garments.

The production of composites with common hydrogel materials as the matrix has proven difficult, since hydrogels usually swell greatly in water and interact poorly with solid components, limiting the load transfer from the fibers to the hydrogel matrix. ${ }^{6}$ The success in fabricating fiber-reinforced soft composites (FRSCs) using charge-balanced PA hydrogels is due to several specific features of the PA gels. ${ }^{6,7}$ Firstly, PA gels have high toughness (fracture energy $\approx 3000 \mathrm{~J} \mathrm{~m}^{-2}$ ) due to energy dissipation by the dynamic ionic bonds. ${ }^{8}$ Secondly, PA gels have self-adjustable ability to adhere to either positively or negatively charged surfaces by forming dynamic ionic bonds to the counter surface, ${ }^{10}$ which results in strong bonding of the PA gel to the glass fibers that have abundant $-\mathrm{SiO}^{-}$groups on the surface. ${ }^{11-13}$ Furthermore, in contrast to traditional hydrogels that swell substantially in water, the as-synthesized PA gels undergo a slight deswelling in water due to the dialysis of small counter ions from the gels, ${ }^{8}$ which prevents significant swelling-induced interfacial mismatching with the stiff fabric.

Fracture energy, the energy per unit area required for a crack to grow in a material, is an intrinsic material parameter used to characterize fracture toughness of the material. One method to evaluate the fracture energy is by performing tearing tests on specimens with a size sufficiently larger than the process zone (i.e., the area surrounding the crack that dissipates energy); the fracture toughness thus obtained is independent of bulk sample size. ${ }^{14-18}$ This holds for traditional gels and elastomers, which have process zones measured in $\mu \mathrm{m}$, or up to a few $\mathrm{mm}$ for the toughest materials. ${ }^{8,15,19-22}$ However, we observed that the fracture energy of the FRSCs, measured by tearing tests, increases with the specimen width even up to the centimeter scale. ${ }^{6}$ Visually, we could observe significant sample deformation during the tearing tests, and the sample failure occurred by fiber pull-out at such sample sizes. ${ }^{7}$ These results suggest that 
our FRSCs have a process zone size larger than the sample sizes used in these observattilonststicle Online and therefore our previously measured fracture energy (i.e., tearing energy) underestimates the intrinsic fracture energy of the soft composites. ${ }^{6,7} \mathrm{We}$ expect that the tearing resistance will increase with sample size until the sample becomes larger than the size of the process zone of the composites, above which the intrinsic fracture energy, independent of sample size, should be observed. Understanding the parameters that control the size of the process zone is important for maximizing the energy dissipation for FRSCs systems.

In this work, we study the sample size dependence of the tearing behaviors for fiberreinforced PA hydrogel composites to determine the process zone size, and to obtain the intrinsic fracture toughness at the saturation of the process zone size. We choose two sets of tough PA hydrogels, P(NaSS-co-DMAEA-Q) and P(NaSS-co-MPTC), formed by random copolymerization of the same anionic monomer, NaSS (sodium $p$-styrenesulfonate), but different cationic monomers, DMAEA-Q (dimethylaminoethylacrylate quaternized ammonium) and MPTC (3-(methacryloylamino)propyl-trimethylammonium chloride) (Fig. S1, ESI†), as model soft matrices. These PA gels, containing $46 \sim 48 \mathrm{wt} \%$ of water, have quite different tensile behaviors. The P(NaSS-co-MPTC) gel has relatively high Young's modulus $(E=2.8 \mathrm{MPa})$, high fracture strength $\left(\sigma_{\mathrm{b}}=3.2 \mathrm{MPa}\right)$ but less fracture strain $\left(\varepsilon_{\mathrm{b}}=8.1 \mathrm{~mm} \mathrm{~mm}^{-1}\right)$ than those of P(NaSS-co-DMAEA-Q) gel $\left(E=0.13 \mathrm{MPa}, \sigma_{\mathrm{b}}=0.95 \mathrm{MPa}, \varepsilon_{\mathrm{b}}=14 \mathrm{~mm} \mathrm{~mm}^{-1}\right)$ (Fig. S2 and Table S1, ESI $\dagger$ ). The two PA gels are both tough, showing comparable tearing fracture energy $\left(G_{\mathrm{c}}=3 \sim 5 \mathrm{~kJ} \mathrm{~m}^{-2}\right.$ ) (Table S1, ESI $\dagger$ ). Two sets of woven glass fabrics with different fiber bundle thicknesses are used (Fig. S3, ESI $\dagger$ ). The thick fabric, denoted as GF, possesses relatively larger fiber bundles (bundle cross-sectional area $A=0.41 \mathrm{~mm}^{2}$ ) and higher fiber bundle fracture force $\left(F_{\mathrm{f}}=375 \mathrm{~N}\right)$ than the thin fabric, denoted as $t$-GF, $\left(A=0.0081 \mathrm{~mm}^{2}\right.$, $\left.F_{\mathrm{f}}=3.6 \mathrm{~N}\right)\left(\right.$ Table S2, ESI $\dagger$ ). The glass fabrics are $10^{3} \sim 10^{4}$ times stiffer than the PA hydrogels (Table S3, ESI†). 
Trouser tearing tests with a sample geometry shown in Fig. 1a are used to evaluatcettheicle Online fracture behavior of the composites at varied sample width, $W$. From this experiment, we experimentally determine the intrinsic fracture resistance and the size of the process zone of the composites. We also elucidate the effects of both the fiber and matrix parameters on the fracture energy and the process zone of the FRSCs. We observe that the intrinsic fracture toughness of the composites is exceptionally high $\left(\approx 1000 \mathrm{~kJ} \mathrm{~m}^{-2}\right)$, along with high tensile strength $(>300$ $\mathrm{MPa}$ ). The high tensile modulus of these composites (several GPa) places them between soft materials and traditional rigid materials. We propose a simple scaling relation for the process zone and discuss the origins of the large energy dissipation.
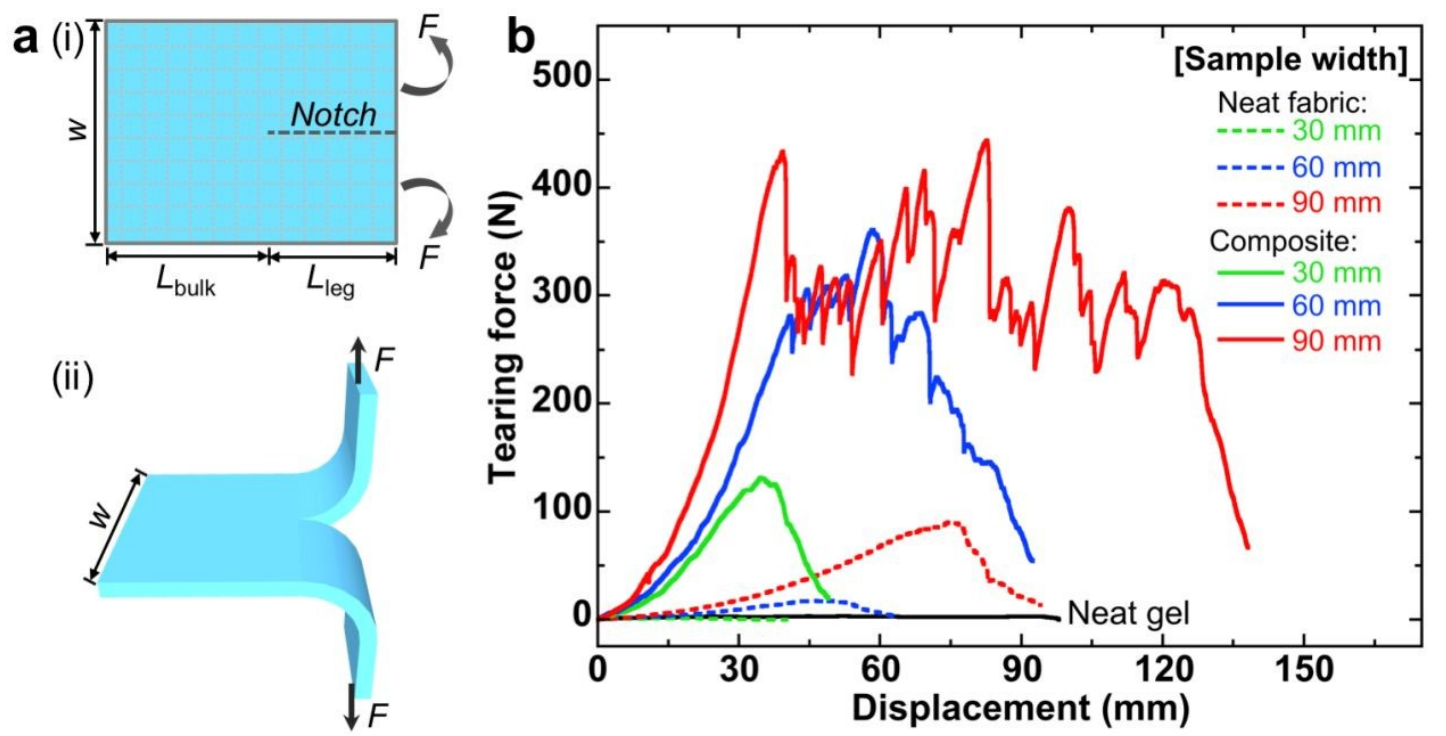

Fig. 1. Results of the tearing tests. a) Illustration of pre-notched sample (i) used for trouser tearing tests and the tearing force direction (ii). The dotted grey lattice in (i) represents the orientation direction of woven fiber bundles. $L_{\mathrm{leg}}=20 \mathrm{~mm} ; L_{\mathrm{bulk}}>W / 2+5 \mathrm{~mm}$. b) Tearing force versus displacement curves for the neat PA gel, neat fabrics, and the PA-GF composites. Three representative sample widths (i.e., $W=30,60$, and $90 \mathrm{~mm}$ ) for both the fabrics and composites were selected. The PA-GF composites are made from the thick glass fabric (GF) and soft P(NaSS-co-DMAEA-Q) hydrogel. 


\section{Results and Discussion}

First, we show the results of FRSCs fabricated from thick glass fabric and relatively soft P(NaSS-co-DMAEA-Q) hydrogel. Fig. 1b depicts the tearing force versus displacement curves for the PA-GF hydrogel composites at representative sample widths ( $W=30,60$, and $90 \mathrm{~mm}$ ). For comparison, the results of the neat woven glass fabric (GF) and neat PA gel components are also shown in the figure. The tearing force of the composites increases dramatically when sample width increases from 30 to $90 \mathrm{~mm}$. Beyond peak force, there is also a change in the shape of the fracture curves. At the width of $30 \mathrm{~mm}$, the curve exhibits a triangular shape, with primarily one peak force. At $60 \mathrm{~mm}$, the curve has a short plateau region where the force maximizes at a value much greater than that of the $30 \mathrm{~mm}$ sample, and a sawtooth pattern emerges. In the $90 \mathrm{~mm}$ wide sample, a long plateau superimposed with a sawtooth pattern exists, with an average plateau force slightly higher than that of the $60 \mathrm{~mm}$ sample. In contrast, the tearing force of the neat GF only exhibits a triangular shape and is much lower than the corresponding composite samples for all the three sample widths, while the tearing force of PA gel is extremely low. The average tearing forces of the composites for the three sample widths are 105,18 , and 4 times the peak tearing forces of the corresponding neat GF, respectively. Based on fracture theory, ${ }^{14,16,18}$ the intrinsic fracture toughness should be independent of sample size when the process zone is smaller than sample dimensions. These results indicate that the intrinsic process zone of the samples discussed here are very large and the observing sample sizes are less than or marginally comparable to the process zone.

To understand the origin of the differences of these curves, we examine the morphologies of the fractured samples with different widths, $W$. These examinations show that fracture behavior is strongly dependent on the fabric width for the composite samples. For samples less than $45 \mathrm{~mm}$ in width, only fiber pull-out is observed (Fig. 2a, (i), (ii)). For the 60 mm sample, we observe a mixture of both fiber pull-out on one surface and fiber fracture on another surface of the fractured pieces (Fig. 2a, (iii)). For $75 \mathrm{~mm}$ and wider samples, fiber 


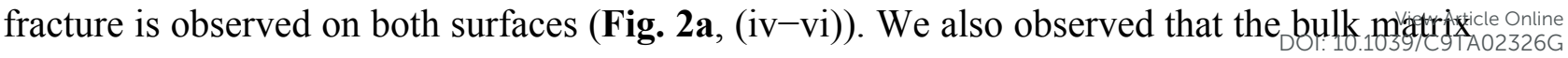
was highly stretched in the composites prior to pull-out or fracture of fiber bundles. Regardless of the change in sample failure mode with sample width, residual gel was clearly observed on the fiber surface, indicating very good adhesion of the gel to the fibers. The gel matrix fractures in all three composite fracture modes (Fig. S4, ESI $\dagger$ ). For the large sample widths $(W \geqslant 60$ $\mathrm{mm}$ ), each force peak in the tearing force versus displacement curves is due to the fracture of a fiber bundle, resulting in the sawtooth pattern (Fig. 1b). Given the strong adhesion of the PA hydrogel on the glass fibers, the main resistance against fiber bundle pull-out comes from the shear stress between the matrix and fibers, and the fracture of a fiber bundle should occur when the total shear force exerted on the bundle by the gel matrix exceeds the tensile fracture strength of the bundle. ${ }^{23-26}$ In contrast, the neat GF used here only shows pull-out of the transverse fiber bundles for all the measured sample widths (Fig. S4, ESI $\dagger$ ), indicating that the total frictional forces exerted on a fiber bundle of the neat fabric are much lower than the fracture strength of the fiber bundle. ${ }^{6,27,28}$

Curves of average peak tearing force, $F_{\mathrm{p}}$, versus sample width, $W$, are shown in Fig. $2 \mathbf{b}$. $F_{\mathrm{p}}$ is determined by the following criterion: for the fracture curve with triangular shape, we choose the peak force as $F_{\mathrm{p}}$; for curves with sawtooth pattern, we determine $F_{\mathrm{p}}$ by averaging the forces between the first and the last peak. We note that the tearing force increases with sample width and reaches a plateau at a characteristic sample width of $75 \mathrm{~mm}$, at which fracture is due entirely to fiber breakage. When the sample width increases beyond $75 \mathrm{~mm}$, the tearing force hardly changes, indicating that the sample width exceeds the process zone size. Tearing energy $(T)$ is calculated from the area under the load versus displacement curves (shown in Fig. 1b) divided by the cross-sectional area of fractured surface..$^{67,29}$ The latter is determined by the sample thickness, $t$, multiplied by the length of the fractured path, $L_{\text {bulk }}$, indicated by green dotted lines in Fig. 2a. As shown in Fig. 2c, the tearing energy also reaches a plateau at the characteristic sample width of $75 \mathrm{~mm}$ for the composites. The neat PA gel, having a process 
zone of only a few millimeters, does not show size-dependent mechanical behaviors atietthercle Online testing condition (sample width $W=7.5 \mathrm{~mm}$ ). ${ }^{7,8,22}$ The neat fabric, which transmits force through friction between the fibers, exhibits a gradual increase in tearing force and energy with increasing sample width over the whole observation range of $30 \sim 110 \mathrm{~mm}$. The result indicates that the process zone size of the neat fabric is larger than $110 \mathrm{~mm}$. This is consistent with the observation that the neat fabric fails by fiber pull-out for the full range of widths in our experiments, due to relatively low frictional shear resistance against the fiber pull-out.
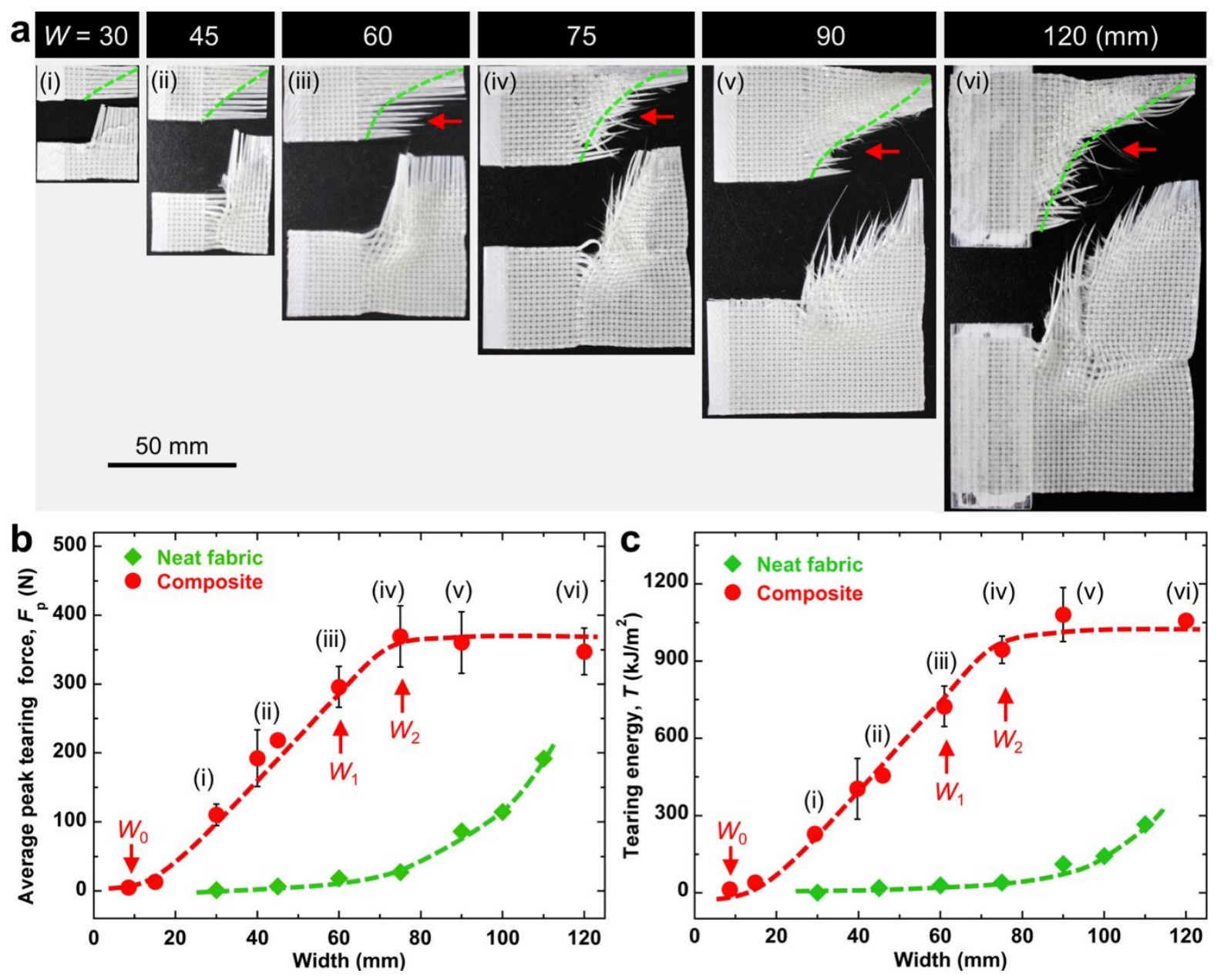

Fig. 2. Size-dependent tearing behavior of the PA-GF hydrogel composites. a) Fracture images of the composites ranging in width of $W=30 \sim 120 \mathrm{~mm}$. The red arrows in (iii-vi) indicate fiber fracture. The green dashed lines in (i-vi) show the fracture path, determined approximately by the fractured hydrogel matrix boundary, which was measured individually for calculating tearing energy. b,c) Average peak tearing force, $F_{\mathrm{p}}(\mathrm{b})$, and tearing energy, $T$ 
(c), versus sample width, $W$, curves for both the neat fabrics and the composites. The numbibefrsicle Online on the plots of the composites correspond to the images of the failure samples in (a). The three characteristic widths of the composites for the failure mode transition, $W_{0}, W_{1}$, and $W_{2}$, are indicated in (b) and (c) by red arrows. Dashed lines in (b) and (c) are drawn as visual guides. The samples are the same as those in Fig. 1b. The error bars represent standard deviations on at least three samples.

Fig. 2b identifies two characteristic sample widths, $W_{1}$ and $W_{2}$, which serve to divide fracture behavior into three regions. In region $\mathrm{I}\left(0<W<W_{1}\right)$ fracture occurred due to fiber pullout, and the tearing force and energy increased with increasing width. Region II ( $\left.W_{1} \leq W<W_{2}\right)$ consisted of a mixed mode fracture of fiber pull-out and the onset of fiber fracture, further increasing the tearing force and energy with increasing width. While fiber fracture begins in region II, pull-out of the fibers can also be seen simultaneously, meaning that the conditions for fiber fracture are not fully established. Finally, in region III $\left(W \geq W_{2}\right)$, fiber fracture was the primary failure mechanism, and no increase in tearing force and energy occurred with increasing width. Fig. 2b, c show that the tearing force and tearing energy increase linearly with sample width until $W_{2}$. Interestingly, there is no change in slope despite the onset of fiber fracture at $W_{1}$. According to fracture theory, ${ }^{14,16,18}$ the tearing energy is an intrinsic material constant, independent of sample size. Here, after reaching region III, when the tearing energy is independent of sample size, the plateau tearing energy $\left(T_{\mathrm{p}}\right)$ can be considered as fracture energy $\left(G_{\mathrm{c}}\right)$ measured by this fracture mode, i.e., $G_{\mathrm{c}}=T_{\mathrm{p}}$. The $G_{\mathrm{c}}$ of the FRSCs reaches $\approx 1000$ $\mathrm{kJ} \mathrm{m}^{-2}$, which is more than 300 times that of the neat PA gel and $\approx 3.5$ times the maximum tearing energy measured for the neat GF $(W=110 \mathrm{~mm})$. The $G_{\mathrm{c}}$ of the fabricated FRSCs outperforms existing tough materials including metals and alloys, ${ }^{30-34}$ exhibiting enhanced fracture resistance. The critical sample width $\left(W_{2}\right)$, at which the tearing force and energy saturate, can be considered as the diameter of the process zone for the FRSCs samples. From 
Fig. 2, we can see, approximately, $W_{1}=60 \mathrm{~mm}$ and $W_{2}=75 \mathrm{~mm}$ for the composites Viryitficle Online $\mathrm{P}(\mathrm{NaSS}-\mathrm{co}$-DMAEA-Q) hydrogel as the matrix. The size of the process zone is on the $\mathrm{cm}$-scale, which is extraordinarily large in comparison to conventional fiber-reinforced polymer composites. ${ }^{35-37}$
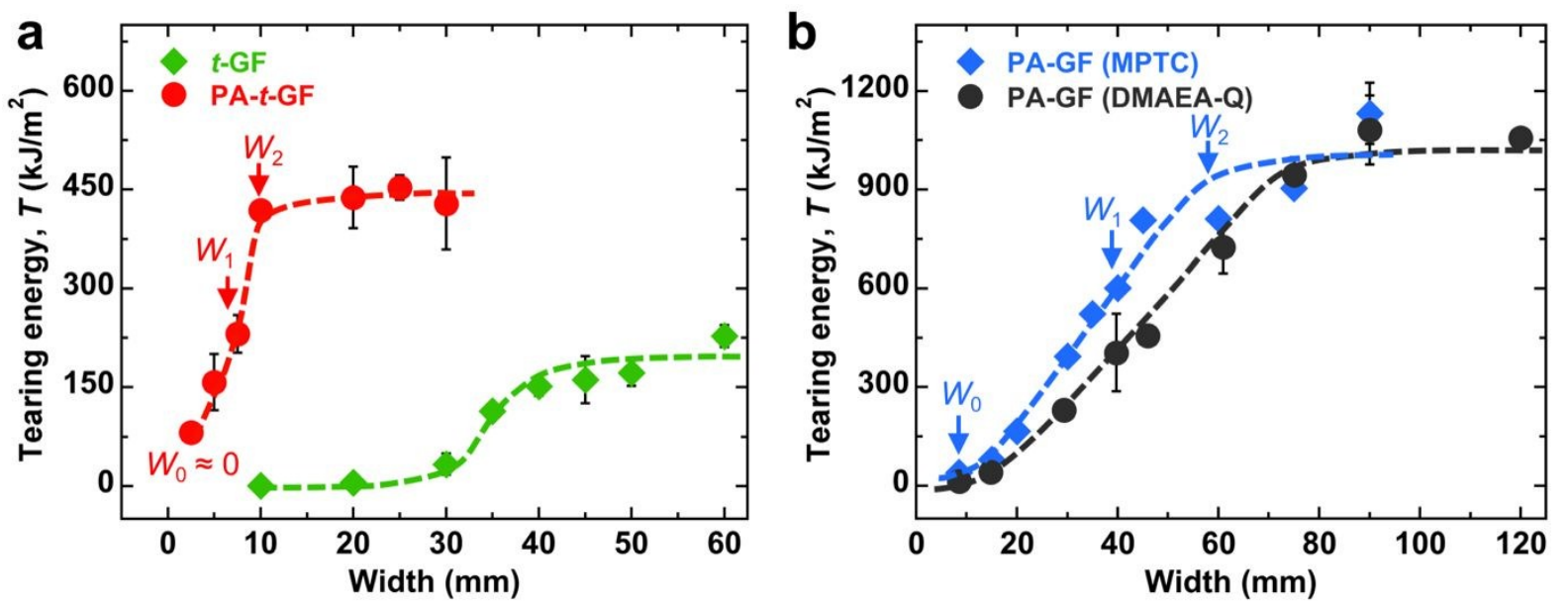

Fig. 3. Tearing energy of FRSCs with different fabrics and hydrogel matrices. a) Tearing energy $(T)$ versus sample width $(W)$ for the thin neat glass fabrics ( $t$-GF) and PA-t-GF hydrogel composites with $\mathrm{P}(\mathrm{NaSS}-\mathrm{co}$-DMAEA-Q) gel as a soft matrix. b) Tearing energy $(T)$ versus sample width $(W)$ for the PA-GF hydrogel composites from the thick glass fabric (GF) and two kinds of PA hydrogels as soft matrices, $\mathrm{P}(\mathrm{NaSS}-\mathrm{co}$-DMAEA-Q) and P(NaSS-co-MPTC). The three characteristic widths of the composites for the failure mode transition, $W_{0}, W_{1}$, and $W_{2}$, are indicated in (a) and (b) by red and blue arrows, respectively. Dashed lines are drawn as visual guides. The error bars represent standard deviation on at least three samples.

To reveal what causes the large process zone in the FRSCs, we studied the effect of geometry and mechanical properties of the fabric and soft matrix. First, we investigated the role of load-bearing capacity of the fiber bundles on the formation of the large process zones of FRSCs. We replaced the thick fabric GF with a thin fabric, $t$-GF, which has fiber bundles that are smaller with lower strength, while the same soft P(NaSS-co-DMAEA-Q) hydrogel was used as the matrix. The composites made from $t$-GF are denoted as PA-t-GF composites. We note 
that PA- $t$-GF composites show the same trend as the PA-GF composites in tearing force (Figicticle Online S5, ESI $\dagger$ ) and tearing energy (Fig. 3a) with changing sample width $W$. The images of the fractured samples (Fig. S6, ESI $\dagger$ ) further show that the PA- $t$-GF composites undergo the same three failure modes as seen in the PA-GF composites (Fig. 2a). The two characteristic sample widths were determined as $W_{1}=7.5 \mathrm{~mm}$ and $W_{2}=10 \mathrm{~mm}$, respectively, for the PA- $t$-GF composites. Both the composites and neat fabrics can achieve a plateau tearing force and tearing energy $G_{\mathrm{c}}$ for the case of thin fabric. This allows us to compare the $G_{\mathrm{c}}$ of the composites to the neat materials. The $G_{\mathrm{c}}$ of the PA-t-GF composites is more than 100 times that of the PA gel and $\approx 2.5$ times that of the neat $t$-GF, clearly demonstrating significant synergistic toughening. Due to good interface adhesion, the critical width $W_{2}$ for the saturation of tearing energy for the composite is reduced to about $1 / 4$ of the neat fabric. Accordingly, the process zone size $L_{\mathrm{p}}$ is 5 $\mathrm{mm}$ and $22.5 \mathrm{~mm}$ for the PA-t-GF composite and the neat $t$-GF fabric, respectively.

Although the PA-t-GF composites exhibit similar fracture behavior (Fig. 2b and Fig. 3a) as those of the PA-GF composites, they show significantly lower maximum tearing force $\left(F_{\mathrm{p}} \approx 12 \mathrm{~N}\right)$ and tearing energy $\left(G_{\mathrm{c}} \approx 430 \mathrm{~kJ} \mathrm{~m}^{-2}\right)$, as well as narrower characteristic widths for the transition modes $\left(W_{1}=7.5 \mathrm{~mm}, W_{2}=10 \mathrm{~mm}\right)$ than those of the PA-GF composites $\left(F_{\mathrm{p}} \approx\right.$ $360 \mathrm{~N}, G_{\mathrm{c}} \approx 1000 \mathrm{~kJ} \mathrm{~m}^{-2}, W_{1}=60 \mathrm{~mm}, W_{2}=75 \mathrm{~mm}$ ). This result indicates that the load-bearing capacity of the fiber bundles plays a key role in determining the process zone size and the $G_{\mathrm{c}}$ of the FRSCs. With a fixed soft matrix, higher load-bearing fiber bundles lead to a larger process zone and a higher fracture force, resulting in a higher $G_{\mathrm{c}}$ in the FRSCs.

To understand the role of the soft matrix in FRSCs, we also studied the tearing behaviors of PA-GF composites with thick glass fabric and P(NaSS-co-MPTC) hydrogels, which has a higher modulus and fracture strength compared to the P(NaSS-co-DMAEA-Q) hydrogels. The raw results are shown in Fig. S7, S8 (ESI $\dagger$ ), and the tearing energy is shown Fig. 3b. For comparison, the result of the PA-GF composites with the relatively soft P(NaSS-co-DMAEAQ) matrix is also shown in Fig. 3b. The two groups of samples are denoted as PA-GF (MPTC) 
and PA-GF (DMAEA-Q), respectively. Similarly, the P(NaSS-co-MPTC) gel can also forimi Aficle Online strong interface with glass fibers and undergo a slight deswelling process in water during dialysis. ${ }^{8}$ While the PA-GF (MPTC) composites also exhibit size-dependent tearing properties and three failure modes (Fig. S7, S8, ESI† ), they show relatively smaller characteristic sample widths $\left(W_{1}=40 \mathrm{~mm}, W_{2}=60 \mathrm{~mm}\right)$, compared to the PA-GF (DMAEA-Q) composites $\left(W_{1}=\right.$ $60 \mathrm{~mm}, W_{2}=75 \mathrm{~mm}$ ). This result indicates that the mechanical properties of the soft matrix are also critical in controlling the process zone size of the FRSCs. The PA-GF (MPTC) composites also exhibit an extraordinarily high $G_{\mathrm{c}}\left(\approx 1000 \mathrm{~kJ} \mathrm{~m}^{-2}\right)$ in the saturated regime (Fig. 3b). The comparison of the two composite groups suggests that, for a fixed reinforcing glass fabric, a stronger and/or higher stiffness soft matrix can help to slightly shift the process zone to a relatively smaller size, allowing the FRSCs to achieve a saturated $G_{\mathrm{c}}$ at a relatively smaller sample width.

To summarize the experiments, the relationship between the tearing energy, $T$, and the fabric width, $W$, is non-linear for both the PA-GF composites and neat fabrics (Fig. 2c and Fig. 3). The $T$ versus $W$ curves can be roughly divided into three regimes: the narrow width regime $\left(0<W<W_{0}\right)$ where $T$ weakly increases with $W$, the intermediate regime $\left(W_{0} \leq W<W_{2}\right)$ where $T$ rapidly increases with $W$, and the plateau regime $\left(W \geq W_{2}\right)$ where $T$ reaches a constant $G_{\mathrm{c}}$, independent of $W$. Here we express the intermediate and plateau regimes, respectively, by Eqs. 1 and 2:

$$
T=k \cdot\left(W-W_{0}\right)+T_{0} \text { for } W_{0} \leq W<W_{2}
$$

and

$$
G_{\mathrm{c}}=k \cdot\left(W_{2}-W_{0}\right)+T_{0} \text { for } W \geq W_{2}
$$

where $k$ is the linearity constant with a dimension of energy density, and $T_{0}$ is the $T$ at $W_{0}$, which is almost negligible comparing to the plateau $T_{\mathrm{p}}=G_{\mathrm{c}}$ for both the FRSCs and neat fabrics. The experimentally determined parameters $\left(W_{0}, W_{1}, W_{2}, G_{\mathrm{c}}\right.$, and $\left.k\right)$ for the three series of FRSCs and the two kinds of neat fabrics used in this study are summarized in Table 1. Comparing with 
the neat fabrics that have a very wide $W_{0}(\sim 30 \mathrm{~mm}$ and $\sim 70 \mathrm{~mm}$ for the thin $t$-GF and thicklificticle Online respectively), the FRSCs have a much narrower $W_{0}(\sim 10 \mathrm{~mm})$ for thick GF composites and a negligible $W_{0}$ for the thin $t$-GF composites.

The three regimes of the fabric width indicate different sample failure mechanisms. For neat fabrics, in the narrow regime $\left(0<W<W_{0}\right)$, the tearing resistance should be related to sliding friction between fiber bundles. In the intermediate regime $\left(W_{0} \leq W<W_{2}\right)$, the interlocking effect due to woven texture of fiber bundles plays dominant role against fiber pull-out, so that the tearing resistance rapidly increases with sample width $W$, leading to fiber breakage starting at $W_{1}$. At $W_{2}$ fibers break entirely and the tearing resistance becomes constant against further increase of $W$. For FRSCs, the strong adhesion between the fiber bundles and the PA hydrogel matrices effectively transmit the force to a large zone so that the samples almost do not show the narrow regime and directly go to the intermediate regime.

Table 1. Characteristic parameters determined by the tearing tests.

\begin{tabular}{ccccccc}
\hline Samples & $\begin{array}{c}\left.W_{0} \mathrm{a}\right) \\
(\mathrm{mm})\end{array}$ & $\begin{array}{c}\left.W_{1} \mathrm{~b}\right) \\
(\mathrm{mm})\end{array}$ & $\begin{array}{c}\left.W_{2} \mathrm{~b}\right) \\
(\mathrm{mm})\end{array}$ & $\begin{array}{c}\left.G_{\mathrm{c}} \mathrm{c}\right) \\
\left(\mathrm{kJ} \mathrm{m}^{-2}\right)\end{array}$ & $\begin{array}{c}k^{\mathrm{d})} \\
\left(\mathrm{MJ} \mathrm{m}^{-3}\right)\end{array}$ & $\begin{array}{c}\left.2 L_{\mathrm{c}} \mathrm{e}\right) \\
(\mathrm{mm})\end{array}$ \\
\hline $\begin{array}{c}\text { PA-GF } \\
(\mathrm{P}(\text { NaSS-co-DMAEA-Q }))\end{array}$ & $\approx 10$ & 60 & 75 & $\approx 1000$ & 15.4 & 184 \\
$\begin{array}{c}\text { PA-GF } \\
(\mathrm{P}(\mathrm{NaSS}-\mathrm{co}-\mathrm{MPTC}))\end{array}$ & $\approx 10$ & 40 & 60 & $\approx 1000$ & 20.0 & 54 \\
$\begin{array}{c}\text { PA-t } t \text { GF } \\
(\mathrm{P}(\mathrm{NaSS}-\mathrm{co}-\mathrm{DMAEA}-\mathrm{Q}))\end{array}$ & $\approx 0$ & 7.5 & 10 & $\approx 430$ & 43.0 & 11 \\
$\begin{array}{c}\text { Neat } t \text {-GF } \\
\text { Neat GF }\end{array}$ & $\approx 30$ & 35 & 45 & $\approx 170$ & 11.3 & - \\
\hline
\end{tabular}

a) $W_{0}$ is the sample width at which the $T$ versus $W$ curves in Fig. 2c and Fig. 3 show inflection in the relatively narrow sample widths.

b) $W_{1}$ and $W_{2}$ are the two characteristic sample widths for the onset of mode transition from fiber pull-out to fracture $\left(W_{1}\right)$ and finishing of mode transition beyond which all the fibers fracture $\left(W_{2}\right)$.

c) $G_{\mathrm{c}}$ is the fracture energy determined by the tearing tests. 
d) $k$ is estimated from the slope of the intermediate regime $\left(W_{0} \leq W<W_{2}\right)$.

e) $L_{\mathrm{c}}$, calculated from Eq. 6, stands for the critical transverse fiber length for the failure mode transition from fiber pull-out to fracture.

f) N/A represents undetectable value up to the sample width $W=110 \mathrm{~mm}$ for the thick GF studied in this experiment.

Next, we investigate what controls the critical widths $W_{1}$ (or $W_{2}$ ) and the linearity constant $k$. First, we discuss the scaling relation between the process zone size and the structural and mechanical parameters of the composites. From our experimental results, we observe that as the fiber bundle strength increases, the process zone widens. On the other hand, with a stronger matrix, the process zone narrows. These results suggest that the process zone size is determined by the competition between the fiber bundle strength and the shear resistance exerted on the fiber against pull-out.

The important characteristics of the FRSCs developed here include: (i) A significant difference in the stiffness and strength between the fabric and matrix. Specifically, the PA gel matrices are $3 \sim 4$ orders of magnitude lower in modulus than the stiff glass fabrics (Table S1, ESI $†$ ). ${ }^{6-8}$ (ii) Good force transmission between the stiff fibers and the soft matrix. The PA gel matrix strongly bonds to the fiber bundles, based on the self-adjustable coulombic adhesion of the PA to the glass fibers. ${ }^{10}$ In addition, the in situ polymerization of the PA gels in the presence of fabrics also ensures good penetration of the gels into the fiber bundles, resulting in an interlocking effect. ${ }^{7,10}$ (iii) High viscoelastic energy dissipation of the PA gels due to dynamic ionic bonds, which also enhances the bonding strength to glass fibers. ${ }^{8,38}$ Given feature (i), the deformation of the rigid fibers is negligible compared to the matrix due to the high compliance of the matrix. Furthermore, feature (ii) indicates good interfacial bonding between the matrix and fiber bundles, and matrix failure always occurs regardless of the fiber failure mode transition. 
While most of the fiber pull-out models are based on an interfacial failure mechativistricle Online that occurs for fibers embedded in comparably stiff matrices, ${ }^{39,40}$ we propose a simple model based on bulk failure of a dissipative soft matrix that occurs in our FRSCs systems. We consider an extremely simplified case of pulling out a straight single fiber bundle from the matrix, without considering any interlocking effect of the transverse fibers as well as the complex change in geometry near the crack front during tearing. With this assumption, the onset of the transition from bundle pull-out to bundle fracture is determined by the condition that the total shear force exerted on a fiber bundle, $F_{\mathrm{s}}$, equals the maximum tensile force of the fiber bundle, $F_{\text {f: }}$

$$
F_{\mathrm{s}}=F_{\mathrm{f}}
$$

For small width, one anticipates that $F_{\mathrm{s}}<F_{\mathrm{f}}$, fiber pull-out as the gel fractures; when $F_{\mathrm{s}} \geq F_{\mathrm{f}}$, both the fiber and gel fracture simultaneously. The total shear force exerted on a transverse fiber bundle, $F_{\mathrm{s}}$, is estimated by:

$$
F_{\mathrm{s}}=\tau_{\mathrm{s}} \cdot S \cdot L \text { for } 0<L<L_{\mathrm{c}}
$$

and

$$
F_{\mathrm{S}}=F_{\mathrm{f}}=\tau_{\mathrm{s}} \cdot S \cdot L_{\mathrm{c}} \text { for } L \geq L_{\mathrm{c}}
$$

where $\tau_{\mathrm{s}}$ and $S$ are the average shear stress exerted on the fiber bundle and the cross-sectional perimeter of a fiber bundle, respectively, and $L_{\mathrm{c}}$ is the critical bundle length for the failure mode transition in the composites. It should be noted that the transverse fiber bundle length $(L)$ in one leg is half of the sample width $(W)$. Then, the $L_{\mathrm{c}}$ for the failure mode transition is:

$$
L_{\mathrm{c}}=\frac{F_{\mathrm{f}}}{S \cdot \tau_{\mathrm{s}}}
$$

Eq. 6 indicates that the critical length for the failure mode transition in FRSCs systems, which is proportional to the size of the process zone, increases with the load-bearing capacity of the fiber bundle and decreases with both the cross-sectional perimeter of the fiber bundle and the shear stress of the soft matrix. Since the fiber bundle pull-out is due to the rupture of the 
hydrogel matrix, the shear stress $\left(\tau_{\mathrm{s}}\right)$ in Eq. 6 is the shear fracture stress of the matrix virime aricle Online confined space. This scaling relation agrees with our observation that the FRSCs fabricated with thicker fiber bundles have larger process zones, while the FRSCs with a stronger matrix have a smaller process zone.

To make a quantitative comparison of the model with the experimental observations, we calculated $L_{\mathrm{c}}$ from Eq. 6, using parameters shown in Table S2 (ESI $\dagger$ ). In the calculation, we assumed that the shear fracture stress $\left(\tau_{\mathrm{s}}\right)$ is the same as the tensile fracture strength of the matrix $\left(\sigma_{\mathrm{b}}\right)$ (Table S1, ESI $\dagger$ ). The calculated values of $2 L_{\mathrm{c}}$ are shown in Table 1. Comparing with the experimentally determined $W_{1}$ (for initiation of fiber fracture) and $W_{2}$ (for fracture mode dominated by fiber fracture), the predicted $2 L_{\mathrm{c}}$ is on the same order but significantly larger than $W_{1}$ and $W_{2}$, especially for the PA-GF composites using the P(NaSS-co-DMAEA-Q) gel as the matrix. Several reasons can be attributed to the discrepancy: (i) the inter-locking between fibers is not considered; (ii) fiber bundles were treated as a solid bundle and the interlocking effect caused by penetration of the matrix into the fiber bundles was not included in the simple model; and (iii) the shear fracture strength of the matrix in the confined space of fibers could be higher than the tensile fracture strength obtained from bulk measurement, which can be speculated from the phenomenon that the force capacity of an adhesive increases with reducing adhesive thickness in lap-shear tests..$^{23-26,39,41}$ All of these reasons should cause a reduction in $L_{\mathrm{c}}$. Nevertheless, the semi-quantitative agreement between the prediction and the observation indicates that our simple fiber pull-out argument captures some essence of the tearing process of FRSCs.

If we assume that the average shear fracture strength of the matrix is the same as the tensile fracture strength $\left(\sigma_{\mathrm{b}}\right)$ of the bulk samples, we obtain the following relation from Eq. 6:

$$
L_{\mathrm{c}}=\frac{A \cdot \sigma_{\mathrm{f}}}{S \cdot \sigma_{\mathrm{b}}}
$$


where $\sigma_{\mathrm{f}}$ and $A$ are the fracture strength and cross-sectional area of a fiber bundle, respectiviedyuticle Online

Eq. 7 shows that the critical length, $L_{\mathrm{c}}$, for the failure mode transition is proportional to the strength ratio of the fiber bundle and the matrix. The proportional coefficient, $A / S$, is determined by the geometry of the fiber bundles (the coefficient is $A / S=R / 2$ for the fiber bundles with circular cross-section of radius $R$ ). In our soft composites, the strength ratio of the fiber bundle to PA hydrogel matrix is very high $\left(\sigma_{\mathrm{f}} / \sigma_{\mathrm{b}}=10^{2} \sim 10^{3}\right)$ (Table S1 and S2, ESI $\uparrow$ ), so that the $L_{\mathrm{c}}$ is $2 \sim 3$ orders of magnitude larger than that of the bundle thickness. This means that the smaller the force transmission of fibers to soft matrix, the larger the process zone.

The experimental results showed that the characteristic sample widths of the neat thin fabric $t$-GF $\left(W_{0}=30 \mathrm{~mm}, W_{1}=35 \mathrm{~mm}, W_{2}=45 \mathrm{~mm}\right)$ are much larger than those of its soft composite $\left(W_{0} \approx 0 \mathrm{~mm}, W_{1}=7.5 \mathrm{~mm}, W_{2}=10 \mathrm{~mm}\right)$. The large characteristic sample widths of neat $t$-GF can be attributed to the low shear resistance, according to Eq. 6. This is reasonable because the shear resistance from frictional stress between fibers in the neat $t$-GF is much lower than the shear resistance from the soft matrix in the composites. Eq. 6 also explains, for the case of neat thick fabric GF, why a much larger narrow regime $\left(W_{0}=70 \mathrm{~mm}\right)$ than that of thin fabric $t$-GF $\left(W_{0}=30 \mathrm{~mm}\right)$ is observed, and why $W_{1}$ and $W_{2}$ of fiber bundle fracture are not observed even up to a sample width $W=110 \mathrm{~mm}$ (Table 1). The quantity $F_{\mathrm{f}} / S$ calculated by Eq. 6 is $87.2 \mathrm{~N} \mathrm{~mm}^{-1}$ for thick fabric bundle, which is more than 10 times larger than that of $t$ GF $\left(F_{\mathrm{f}} / S=5.3 \mathrm{~N} \mathrm{~mm}^{-1}\right)$ (Table S2, ESI $\left.\dagger\right)$. Accordingly, the characteristic sample widths $\left(W_{1}\right.$, $W_{2}$ ) of the thick GF should be much larger than those of the $t$-GF.

Next, we discuss what determines the linearity constant $k$. As $k$ has dimensions of energy density, we suggest that it is related to the dissipation energy density in the process zone. If we assume that the interface between the fiber and the gel matrix does not break, the energy dissipation can originate from two possible sources: one is from the matrix and the other is from the fabric. Thus, we make a comparison of $k$ with the work of extension at tensile fracture $\left(W_{\text {ext }}\right)$ for the P(NaSS-co-DMAEA-Q) gel, neat fabric (GF and $t$-GF), and their composites. $W_{\text {ext }}$ was 


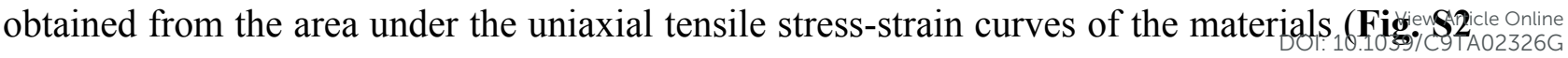
and S9, ESI $\dagger$ ), and the results are summarized in Table S1 for gels and Table S3 for neat fabrics and composites (ESI $\dagger$ ). We see that $W_{\text {ext }}$ of the P(NaSS-co-DMAEA-Q) gel and fabrics are on the same order of magnitude $\left(4 \sim 8 \mathrm{MJ} \mathrm{m}^{-3}\right)$, while the composites have a higher $W_{\text {ext }}$ than those of the individual components $\left(15 \sim 18 \mathrm{MJ} \mathrm{m}^{-3}\right)$. Comparing with the results in Table $1, k$ of the components is on the same order of magnitude with their corresponding $W_{\text {ext }}$. This suggests that the energy dissipation of the composites originates from both the matrix and fibers. It seems to be counter intuitive that the same $k$ applies for the non-fracture regime $\left(W_{0} \leq W<W_{1}\right)$, partially fracture regime $\left(W_{1} \leq W<W_{2}\right)$, and the entire facture regime $\left(W \geq W_{2}\right)$ of fiber bundles. This can be explained by the fact that there is elastic energy stored in fiber bundles and matrix even though the fiber bundles do not break, and this stored energy is dissipated during fiber pull-out. For the neat $t$-GF, $k$ is also very close to its $W_{\text {ext }}$. Although the neat fabrics have larger process zone than the corresponding soft composites, the stored elastic energy is small. As a result, the neat fabrics have lower $G_{\mathrm{c}}$ than the corresponding soft composites.

Therefore, we see that the extraordinarily high toughness $\left(G_{\mathrm{c}}\right)$ of the FRSCs is attributed to their very large process zone, and a large amount of energy dissipation in the process zone due to the presence of tough PA gels. In addition, large process zone increases the energy loss due to fiber breakage. The proposed simple fracture model provides a good guide for designing FRSCs with large process zone sizes and superior fracture resistance. To optimize energy dissipation, a combination of thin but strong fiber bundles with a soft and energy dissipative matrix should be chosen. Furthermore, high fiber density is favored to achieve high shear resistance..$^{23-26,39,41}$ 


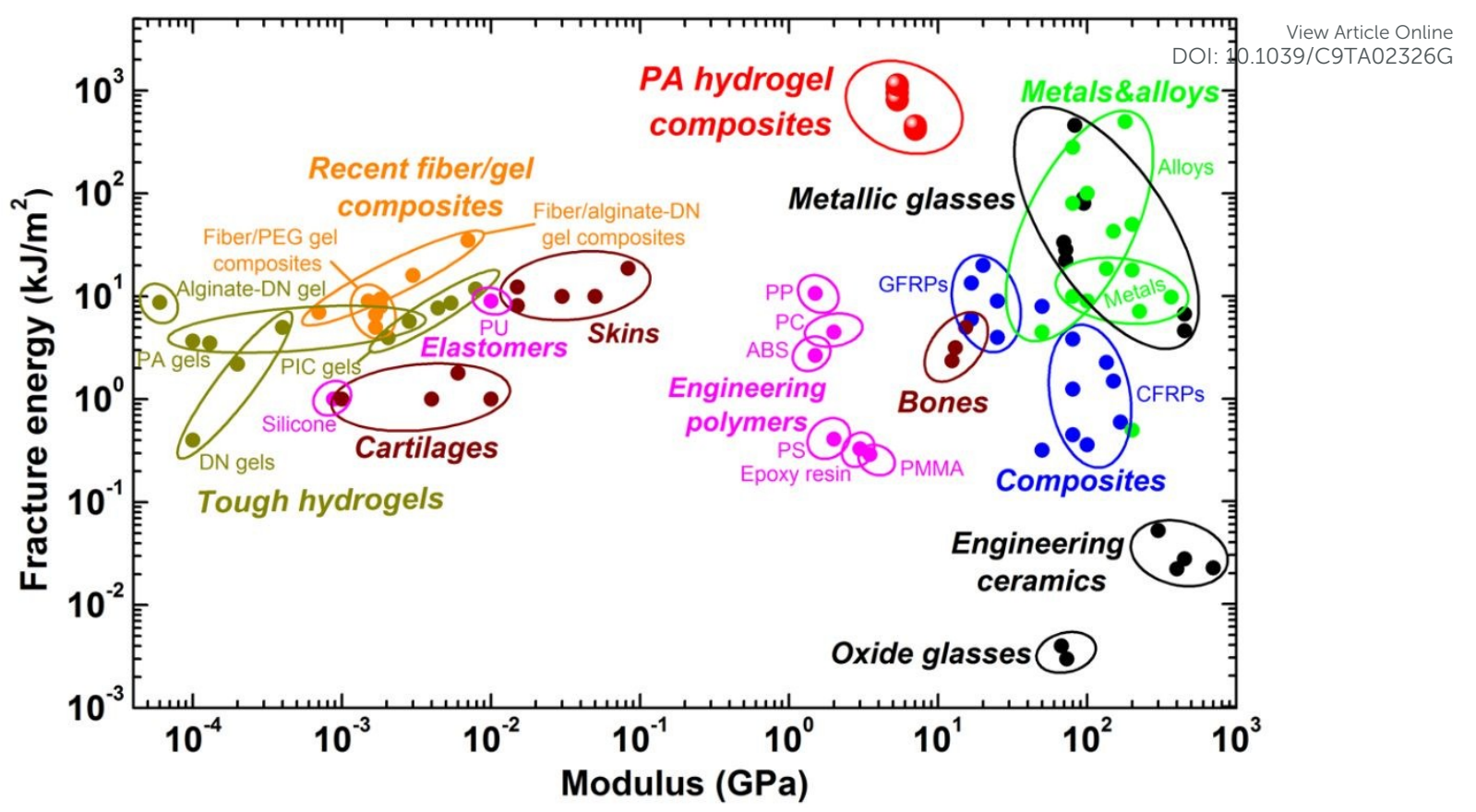

Fig. 4. Fracture energy versus Young's modulus map of materials. The selected materials include oxide glasses, ${ }^{42}$ engineering ceramics, ${ }^{30,43}$ tough metallic glasses, ${ }^{31-33}$ traditional composites (including GFRPs and CFRPs), ${ }^{30}$ metals and alloys, ${ }^{30,34}$ engineering polymers (including commercially available elastomers), ${ }^{30}$ tough hydrogels, $, 8,15,44,45$ natural tissues, ${ }^{46,47}$ recent reported fiber/hydrogel composites, ${ }^{48,49}$ and tough PA hydrogel composites (i.e., the FRSCs) in this work. Despite containing hydrated biocompatible hydrogels, the developed FRSCs show outstanding mechanical properties, allowing them to fill the gap between soft materials and traditional rigid materials.

The fabricated FRSCs are flexible, but they possess very high tensile modulus (several $\mathrm{GPa})$ and tensile strength $(>300 \mathrm{MPa})$, due to the presence of glass fibers in the aligned direction (Fig. S9 and Table S3, ESI $\dagger$ ). This results in composites which possess mechanical properties significantly different from traditional hydrogels, and in some cases similar to structural materials such as metals. To compare our materials to both soft materials as well as structural materials, we show a fracture energy $G_{\mathrm{c}}$ versus Young's modulus map for a wide range of materials in Fig. 4. We can see that the hydrogel-based FRSCs presented here are 
much stronger and tougher than even the best hydrogel materials, giving them strong potelintiaflicle Online as structural biomaterials. Surprisingly, the $G_{\mathrm{c}}$ of the FRSCs is not only higher than traditional FRPs from the combination of hard fibers and hard plastics, ${ }^{2}$ but also outperforms some hard materials, including metals and alloys, demonstrating that they fill the gap between soft materials and traditional rigid materials. From the relation shown in Eq. 7, we speculate that traditional FRPs from hard-hard combinations have much smaller process zones than the FRSCs. Furthermore, the hard matrix of FRPs usually has poor energy dissipation. As a result, traditional FRPs show lower fracture resistance in comparison to the soft composites from tough hydrogels.

\section{Conclusions}

In this work, we show that the fracture mode and tearing resistance of FRSCs are strongly dependent on the sample width even up to a scale as large as cm-order. Two characteristic sample widths, $W_{1}$ and $W_{2}$, where $W_{2}=1.3 \sim 1.5 W_{1}$, are observed. With increasing sample width, $W$, the fracture mode changes from fiber pull-out mode at $0<W<W_{1}$, to mixed mode of fiber pull-out and fracture at width in $W_{1} \leqslant W<W_{2}$, and then to all fiber fracture mode at $W \geqslant W_{2}$. The tearing energy linearly increases with sample width $W$ and saturates at $W \geqslant W_{2}$, giving the intrinsic fracture energy $G_{\mathrm{c}}$ of the composites. $W_{1}$ and $W_{2}$, and $G_{\mathrm{c}}$ increase with load-bearing capacity of the fiber bundle. Using thick fiber bundles that have high fracture strength, the $G_{\mathrm{c}}$ reaches an exceptionally high value of $\sim 1000 \mathrm{~kJ} \mathrm{~m}^{-2}$. Both higher load-bearing capacity of the rigid fabric and softer matrix result in a larger process zone, which causes the increased fracture resistance of FRSCs. Based on the competition between fiber strength and matrix strength, we propose a simple model for the critical transverse fiber length, $L_{\mathrm{c}}$, to achieve fiber fracture. The model shows that the large strength ratio of the fiber bundle to the soft matrix leads to the large process zone. The $2 L_{\mathrm{c}}$ from the model are on the same order with $W_{1}$ and $W_{2}$. A more accurate model considering the complex deformation of the composites during the tearing tests is required in future work. To design super-tough FRSCs, a combination of rigid 
fabrics from high strength fibers and soft and tough matrices that dissipate large amoutif offle Online $^{\text {am }}$ energy, and a strong bonding between the rigid fabrics and the soft hydrogel matrix are essential. The optimized mechanical performance allows the hydrogel-based FRSCs to fill the gap between soft materials and traditional rigid materials.

\section{Experimental Section}

Materials: Dimethylaminoethylacrylate quaternized ammonium (DMAEA-Q, $79 \mathrm{wt} \%$, cationic monomer) was supplied by MT Aqua Polymer, Inc., Japan. Sodium $p$-styrenesulfonate (NaSS, anionic monomer), 3-(methacryloylamino)propyl-trimethylammonium chloride (MPTC, cationic monomer), $N, N$ '-methylene-bis-acrylamide (MBAA, chemical cross-linker), and $\alpha$-ketoglutaric acid ( $\alpha$-keto, photoinitiator) were purchased from Wako Pure Chemical Industries, Ltd., Japan. The chemical structures of the monomers and cross-linker are shown in Fig. S1 (ESI $\dagger$ ). All reagents were used as received, and Milli-Q (18.3 M $\Omega$ ) water was used in all experiments. The mechanical properties of the two PA hydrogels are shown in Fig. S2 and Table S1 (ESI $\dagger)$

The glass fabrics are woven from fiber bundles, and each bundle consists of a number of fibers (Fig. S10 and S11, ESI†). Two kinds of plain weave glass fiber fabrics from E-glass were used as received. One is thick (denoted as GF, surface density $\sigma_{\mathrm{a}}=590 \mathrm{~g} \mathrm{~m}^{-2}$, thickness $t$ $=0.59 \mathrm{~mm}$, mesh size $h \approx 0.40 \mathrm{~mm}$, single fiber radius $r=7 \mu \mathrm{m})$, purchased from Marukatsu Co., Ltd., Japan. The other is thin (denoted as $t$-GF, $\sigma_{\mathrm{a}}=47 \mathrm{~g} \mathrm{~m}^{-2}, t=0.045 \mathrm{~mm}, h \approx 0.14 \mathrm{~mm}$, $r=2.5 \mu \mathrm{m}$ ), supplied by Asahi Kasei Co., Ltd., Japan. The cross-section of the fiber bundles was observed by the SEM micrographs of the composites, and the cross-sectional perimeter $S$ $=4.3 \pm 0.2 \mathrm{~mm}$ for GF; $S=0.68 \pm 0.03 \mathrm{~mm}$ for $t$-GF (Fig. S10 and S11, ESI $\dagger$ ). The fabric mesh sizes were measured by ImageJ software from the graphs of the composite samples $(N>$ 5). The morphologies and physical parameters of the two kinds of fabrics are presented in Fig. S3 and Table S2 (ESI $\dagger$ ). The glass fabrics have a high wettability to water. ${ }^{7}$ 
Fabrication of FRSCs: Polyampholyte-woven glass fabric (PA-GF) hydrogel compojsitessicle Online were fabricated via a facile in situ radical polymerization, as described previously. ${ }^{7}$ Typically, for preparing PA-GF composites, the precursor solution containing anionic monomer (NaSS), cationic monomer (DMAEA-Q), crosslinker (MBAA), and photoinitiator ( $\alpha$-keto) was prepared according to the previous studies. ${ }^{8,10,38}$ The total ionic monomer concentration and the molar fraction of anionic monomer were fixed as $2.5 \mathrm{~mol} \mathrm{~L}^{-1}$ and 0.52 , respectively, and the molar fractions of both crosslinker and initiator were $0.10 \mathrm{~mol} \%$, relative to the total monomer concentration. The precursor solution was injected into a reaction cell consisting of a pair of glass plates as walls. In between, a sheet-like woven GF was embedded in the center by using two silicone spacers with $0.5 \mathrm{~mm}$ thickness. The reaction cell was irradiated with $365 \mathrm{~nm}$ ultraviolet lamp for 11 hours at ambient temperature under an argon atmosphere to complete the polymerization. After polymerization, the as-prepared hydrogel composites (denoted as PAGF (DMAEA-Q)), $\approx 1.5 \mathrm{~mm}$ thickness, were obtained, and then immersed in adequate deionized water for at least five days to remove residual chemicals until the deswelling equilibrium was achieved. During this process, mobile counter-ions were dialyzed gradually, and the oppositely charged ions on the polymer chains formed dynamic ionic bonds through electrostatic attraction, and the interfacial bonding between PA and GF was also enhanced accordingly. Another cationic monomer, MPTC, was also used instead of DMAEA-Q to fabricate PA hydrogel composites (denoted as PA-GF (MPTC)). PA- $t$-GF hydrogel composites were fabricated using thin woven glass fabric $(t-\mathrm{GF})$ by the same procedure but using $0.2 \mathrm{~mm}$ thick silicone spacers. The thicknesses of the silicone spacers were chosen above which the hydrogel layers are thick enough so that the tensile and tearing behaviors of the composites became independent of the spacer thickness. ${ }^{6,7}$ In addition, neat PA hydrogels were also prepared by this procedure (1.5 mm spacer), without the GF sheet embedded.

Other experimental details are presented in the Supplementary Information. 
This research was supported by JSPS KAKENHI Grant Numbers JP17H06144, JP17H06376, and by ImPACT Program of Council for Science, Technology and Innovation (Cabinet Office, Government of Japan). D.R.K. thanks the Hokkaido University Tenure Track System Promotion Program for partial funding. Y.H. thanks MEXT, Japan for the scholarship during his PhD study. Institute for Chemical Reaction Design and Discovery (ICReDD) was established by World Premier International Research Initiative (WPI), MEXT, Japan. Authors thank Dr. Tsuneaki Tanabe from Asahi Kasei Co., Ltd., Japan for providing the thin glass fabric.

\section{References}

1 U. G. K. Wegst, H. Bai, E. Saiz, A. P. Tomsia and R. O. Ritchie, Nat. Mater., 2015, 14, $23-36$.

2 P.K. Mallick, Fiber-Reinforced Composites: Materials, Manufacturing, and Design, 2007, vol. 19.

3 M. P. E. Wenger, L. Bozec, M. A. Horton and P. Mesquida, Biophys. J., 2007, 93, 12551263.

4 G. A. Ateshian and V. C. Mow, in Friction, lubrication, and wear of articular cartilage and diarthrodial joints, 2005, pp. 447-494.

5 W. Yang, V. R. Sherman, B. Gludovatz, E. Schaible, P. Stewart, R. O. Ritchie and M. A. Meyers, Nat. Commun., 2015, 6, 6649.

6 D. R. King, T. L. Sun, Y. Huang, T. Kurokawa, T. Nonoyama, A. J. Crosby and J. P. Gong, Mater. Horiz., 2015, 2, 584-591.

7 Y. Huang, D. R. King, T. L. Sun, T. Nonoyama, T. Kurokawa, T. Nakajima and J. P. Gong, Adv. Funct. Mater., 2017, 27, 1605350.

8 T. L. Sun, T. Kurokawa, S. Kuroda, A. Bin Ihsan, T. Akasaki, K. Sato, M. A. Haque, T. Nakajima and J. P. Gong, Nat. Mater., 2013, 12, 932-937.

9 S. K. N. Kutty and G. B. Nando, J. Appl. Polym. Sci., 1991, 43, 1913-1923. 


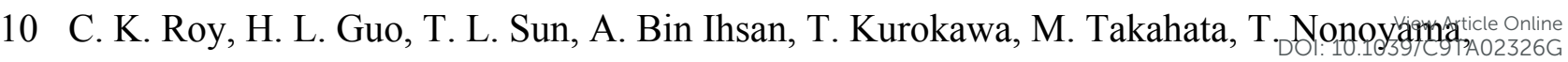
T. Nakajima and J. P. Gong, Adv. Mater., 2015, 27, 7344-7348.

11 F. S. Lameiras, A. L. de Souza, V. A. R. de Melo, E. H. M. Nunes and I. D. Braga, Mater. Res., 2008, 11, 217-219.

12 H. Abe, Y. Hara, S. Maeda and S. Hashimoto, J. Phys. Chem. B, 2014, 118, 2518-2522.

13 J. Ahmed, H. Guo, T. Yamamoto, T. Kurokawa, M. Takahata, T. Nakajima and J. P. Gong, Macromolecules, 2014, 47, 3101-3107.

14 P. G. de Gennes, Langmuir, 1996, 12, 4497-4500.

15 J.-Y. Sun, X. Zhao, W. R. K. Illeperuma, O. Chaudhuri, K. H. Oh, D. J. Mooney, J. J. Vlassak and Z. Suo, Nature, 2012, 489, 133-136.

16 X. Zhao, Soft Matter, 2014, 10, 672-687.

17 T. Zhang, S. Lin, H. Yuk and X. Zhao, Extrem. Mech. Lett., 2015, 4, 1-8.

18 G. J. Lake and A. G. Thomas, Proc. R. Soc. A Math. Phys. Eng. Sci., 1967, 300, 108-119.

19 J. P. Gong, Y. Katsuyama, T. Kurokawa and Y. Osada, Adv. Mater., 2003, 15, 1155-1158.

20 J. P. Gong, Soft Matter, 2010, 6, 2583.

21 E. Ducrot, Y. Chen, M. Bulters, R. P. Sijbesma and C. Creton, Science, 2014, 344, 186189.

22 F. Luo, T. L. Sun, T. Nakajima, T. Kurokawa, Y. Zhao, A. Bin Ihsan, H. L. Guo, X. F. Li and J. P. Gong, Macromolecules, 2014, 47, 6037-6046.

23 L. F. M. da Silva, T. N. S. S. Rodrigues, M. A. V Figueiredo, M. F. S. F. de Moura and J. A. G. Chousal, J. Adhes., 2006, 82, 1091-1115.

24 M. D. Banea, L. F. M. Da Silva and R. D. S. G. Campilho, J. Adhes., 2014, 91, 331-346.

25 Y. Boutar, S. Naïmi, S. Mezlini, L. F. M. Da Silva, M. Hamdaoui and M. Ben Sik Ali, J. Adhes. Sci. Technol., 2016, 30, 1913-1929.

26 M. D. Bartlett, A. B. Croll and A. J. Crosby, Adv. Funct. Mater., 2012, 22, 4985-4992.

27 W. A. Scelzo, S. Backer and M. C. Boyce, Text. Res. J., 1994, 64, 291-304. 
28 W. A. Scelzo, S. Backer and M. C. Boyce, Text. Res. J., 1994, 64, 321-329.

View Article Online DOI: 10.1039/C9TA02326G

29 E. Triki, P. Dolez and T. Vu-Khanh, Compos. Part B Eng., 2011, 42, 1851-1859.

30 M. F. Ashby, Materials Selection in Mechanical Design Third Edition, MRS Bull, 2005.

31 J. Schroers and W. L. Johnson, Phys. Rev. Lett., 2004, 93, 20-23.

32 M. D. Demetriou, G. Kaltenboeck, J. Y. Suh, G. Garrett, M. Floyd, C. Crewdson, D. C. Hofmann, H. Kozachkov, A. Wiest, J. P. Schramm and W. L. Johnson, Appl. Phys. Lett., $2009,95,23-26$.

33 M. D. Demetriou, M. E. Launey, G. Garrett, J. P. Schramm, D. C. Hofmann, W. L. Johnson and R. O. Ritchie, Nat. Mater., 2011, 10, 123-128.

34 B. Gludovatz, A. Hohenwarter, D. Catoor, E. H. Chang, E. P. George and R. O. Ritchie, Science, 2014, 345, 1153-1158.

35 S. C. Tjong, S.-A. Xua, R. K.-Y. Li and Y.-W. Mai, Compos. Sci. Technol., 2002, 62, 2017-2027.

36 D. A. Norman and R. E. Robertson, J. Appl. Polym. Sci., 2003, 90, 2740-2751.

37 Q. Liu and M. Hughes, Compos. Part A Appl. Sci. Manuf., 2008, 39, 1644-1652.

38 A. Bin Ihsan, T. L. Sun, S. Kuroda, M. A. Haque, T. Kurokawa, T. Nakajima and J. P. Gong, J. Mater. Chem. B, 2013, 1, 4555-4562.

39 A. Takaku and R. G. C. Arridge, J. Phys. D. Appl. Phys., 1973, 6, 310.

40 A. Kelly and W. R. Tyson, J. Mech. Phys. Solids, 1965, 13, 329-350.

41 H. L. Cox, Br. J. Appl. Phys., 1952, 3, 72-79.

42 R. J. Wang, F. Y. Li, J. F. Wang and W. H. Wang, Appl. Phys. Lett., 2003, 83, 2814-2816.

43 W. J. Clegg, K. Kendall, N. M. Alford, T. W. Button and J. D. Birchall, Nature, 1990, 347, $455-457$.

44 S. Naficy, H. R. Brown, J. M. Razal, G. M. Spinks and P. G. Whitten, Aust. J. Chem., 2011, 64, 1007. 


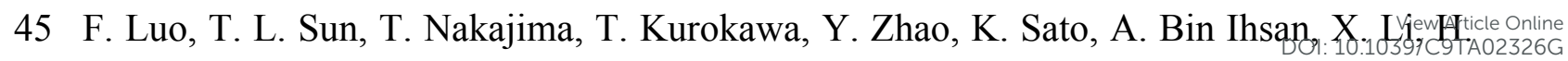
Guo and J. P. Gong, Adv. Mater., 2015, 27, 2722-2727.

46 U. G. K. Wegst and M. F. Ashby, Philos. Mag., 2004, 21, 2167-2181.

47 J. Li, Z. Suo and J. J. Vlassak, J. Mater. Chem. B, 2014, 2, 6708-6713.

48 A. Agrawal, N. Rahbar and P. D. Calvert, Acta Biomater., 2013, 9, 5313-5318.

49 S. Lin, C. Cao, Q. Wang, M. Gonzalez, J. E. Dolbow and X. Zhao, Soft Matter, 2014, 10, $7519-7527$. 
Table of Contents Entry:

Fiber-reinforced polyampholyte hydrogels have demonstrated superior fracture resistance by saturating extraordinarily large energy-dissipative process zones, outperforming other existing tough materials.

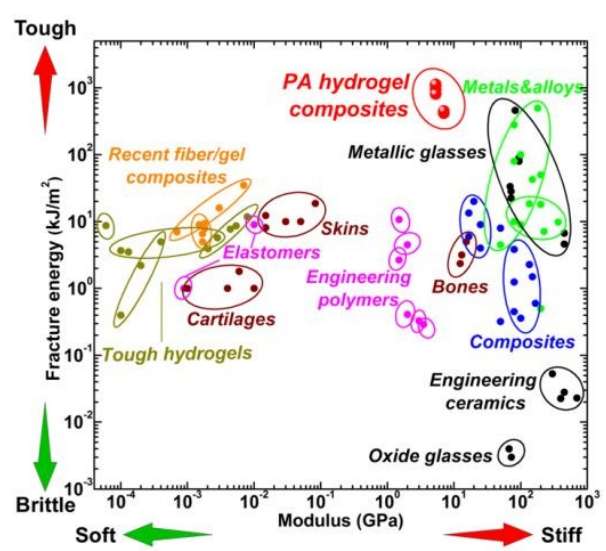

(ToC Figure) 